\title{
Nasopharyngeal swabs during SARS-CoV-2 pandemic: a role for the otolaryngologist
}

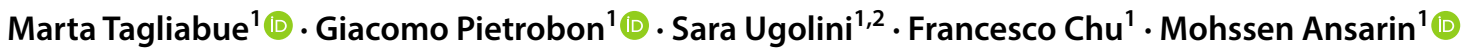

Received: 22 April 2020 / Accepted: 27 April 2020 / Published online: 7 May 2020

c) Springer-Verlag GmbH Germany, part of Springer Nature 2020

To the Editor

As of 19th April 2020, more than 2,200,000 cases of Severe Acute Respiratory Syndrome Coronavirus 2 (SARS-CoV-2) and more than 152,000 related deaths have been confirmed worldwide in 213 countries [1].

SARS-CoV-2 has proven to be highly contagious, but its fatality rate varies among countries and remains ultimately unclear. Strict isolation is the common policy of governments to contain further spread of the disease. The role of asymptomatic or paucisymptomatic patients is still under investigation, but it may explain the diffusion of the virus [2].

For the diagnosis in outpatients, the United States Centers for Disease Control and Prevention recommend Reverse Transcription Polymerase Chain Reaction (RT-PCR) testing on nasopharyngeal swabs, which have higher yields than nasal or throat swabs [3]. RT-PCR testing has substituted viral cultures as gold-standard because of the high accuracy and better timing [3]. Nasopharyngeal swabs are considered easy and quick to collect, they are not time-consuming and they can be performed anywhere, even to people sitting in a car. Bronchoalveolar lavage is considered more sensitive than nasopharyngeal specimens, with a sensitivity around $97 \%$, but its use is limited to critically ill patients on mechanical ventilation. More recently, serologic tests have to gain attention for their potential of screening wider populations, identifying uninfected and/or healed subjects and thus directing future policies. So far, though, they are still under investigation and their current role is, therefore, limited [2].

Giacomo Pietrobon

giacomo.pietrobon@gmail.com

1 Division of Otolaryngology and Head and Neck Surgery, European Institute of Oncology IRCCS, Via Ripamonti 435, Milan, Italy

2 Department of Otorhinolaryngology, University of Pavia, IRCCS Fondazione Policlinico San Matteo, Pavia, Italy
The false-negative rates of RT-PCR is reported to be around 51.6\% [4]. Reaching the nasopharynx with a swab is not easy: the intranasal anatomy is highly variable (e.g. hypertrophic inferior turbinates, deviated septum, conchae bullosae, synechiae) and this factor, coupled with patients' discomfort, may result in a flawed sampling of the nasal cavity, the inferior turbinate or the middle meatus instead. We hypothesize that part of these limited results may be linked to a wrong diagnostic procedure performed collecting the swabs.

The technique for the correct sampling of the nasopharynx was shown in 2009 during the swine flu epidemic and was further updated in a recent report dedicated to the SARS-CoV-2 pandemic [5]. Formal educational programs already exist because of previous epidemics, such as the Middle Eastern Respiratory Syndrome Coronavirus [6], but on many iconographic materials one can often see swabs positioned vertically or introduced into the nasal cavity only for few centimeters, thus potentially ending up in an inaccurate procedure. It would be invaluable to train healthcare personnel with the aid of a live endonasal endoscopic demonstration of nasal and nasopharyngeal anatomy and sampling, and in this field the active role of the otolaryngologist becomes very relevant (Fig. 1).

In our Institution, since the outbreak of the infection, we experienced a very strict collaboration between the otolaryngologist and the personnel dedicated to the swab collection. The latter benefited from observing the endoscopic anatomy of the patient and interacting with the otolaryngologist during the examination so that they were eventually able to acquire major confidence with the procedure if compared to the lone description or the passive observation of a video. Even patients referred less discomfort during the sampling procedure and were obviously more compliant.

Much of the current and future outcomes about COVID 19 will depend on the actions undertaken from now on. Even a simple diagnostic test, such as a nasopharyngeal swab, has a great impact on the management of the 


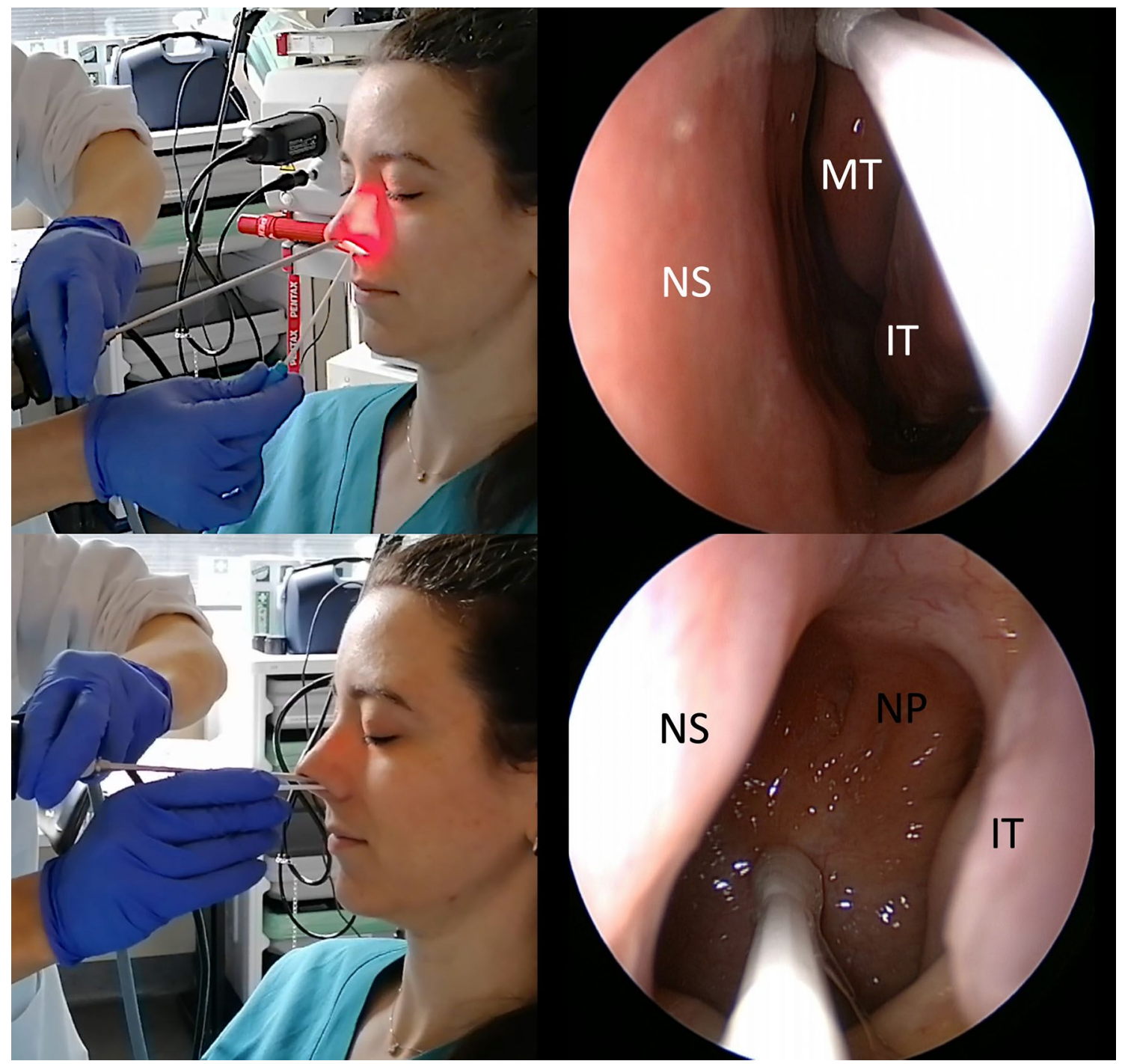

Fig. 1 Demonstration of different sampling sites aided by endonasal endoscopy (Karl Storz $4 \mathrm{~mm} 0^{\circ}$ lens). When inserted parallel the external nasal profile, the swab samples the vault of the nasal fossa or the middle meatus (upper half). To reach the nasopharynx, the swab needs to be inserted parallel to the floor of the nasal fossa and pushed all the way in until blocked by the posterior wall of the nasopharynx (lower half). NS nasal septum, IT inferior turbinate, MT middle turbinate, NP nasopharynx. Photo source: Department of Otolaryngology and Head and Neck Surgery, European Institute of Oncology, IRCCS. Milan, Italy pandemic and control of the spread of disease. In our experience, because of their being familiar with the upper respiratory airways, the Otolaryngologists should play an active role in training and on-field supervision, especially in the case of troublesome nasopharyngeal samplings.

Acknowledgements We would like to thank Dr Susanna Chiocca for support and idea sharing.

Funding This work was partially supported by the Italian Ministry of Health with Ricerca Corrente and $5 \times 1000$ funds.

\section{Compliance with ethical standards}

Conflict of interest No conflict of interest in this work for all authors.

\section{References}

1. World Health Organization, Coronavirus disease 2019 (COVID 2019) Situation Report-90, https://www.who.int/docs/defaultsource/coronaviruse/situation-reports/20200419-sitrep-90-covid -19.pdf?sfvrsn=551d47fd_4. Accessed 21Apr 2020 
2. Cheng MP, Papenburg J, Desjardins M et al (2020) Diagnostic testing for severe acute respiratory syndrome-related coronavirus-2: a narrative review. Ann Intern Med. https://doi.org/10.7326/ M20-1301 (Epub ahead of print 13 April 2020)

3. Centers for Disease Control and Prevention, Interim Guidelines for Collecting, Handling, and Testing Clinical Specimens from Persons for Coronavirus Disease 2019 (COVID-19). https://www. cdc.gov/coronavirus/2019-ncov/lab/guidelines-clinical-speci mens.html. Accessed 18 Apr 2020

4. Yang Y, Yang M, Shen $\mathrm{C}$ at al. Evaluating the accuracy of different respiratory specimens in the laboratory diagnosis and monitoring the viral shedding of 2019-nCoV infections. 2020. https://doi. org/10.1101/2020.02.11.20021493 (Epub ahead of print)
5. Marty FM, Chen K, Verrill KA (2020) How to obtain a nasopharyngeal swab specimen. NEJM. https://doi.org/10.1056/ NEJMvem2010260 (Epub ahead of print)

6. Al-Tawfiq JA, Rothwell S, Mcgregor HA, Khouri ZA (2018) A multi-faceted approach of a nursing led education in response to MERS-CoV infection. J Infect Public Health 11(2):260-264

Publisher's Note Springer Nature remains neutral with regard to jurisdictional claims in published maps and institutional affiliations. 